\title{
Two Dimensional Ice from First Principles: Structures and Phase Transitions
}

\author{
Ji Chen, ${ }^{1,2, *}$ Georg Schusteritsch, ${ }^{2,3,4}$ Chris J. Pickard, ${ }^{2,3,4}$ Christoph G. Salzmann, ${ }^{5}$ and Angelos Michaelides ${ }^{1,2,3, \dagger}$ \\ ${ }^{1}$ London Centre for Nanotechnology, 17-19 Gordon Street, London WC1H OAH, United Kingdom \\ ${ }^{2}$ Thomas Young Centre, University College London, 20 Gordon Street, London WC1H OAJ, United Kingdom \\ ${ }^{3}$ Department of Physics and Astronomy, University College London, Gower Street, London WC1E 6BT, United Kingdom \\ ${ }^{4}$ Department of Materials Science and Metallurgy, University of Cambridge, \\ 27 Charles Babbage Road, Cambridge CB3 OFS, United Kingdom \\ ${ }^{5}$ Department of Chemistry, University College London, 20 Gordon Street, London WC1H OAJ, United Kingdom
}

(Received 18 August 2015; revised manuscript received 14 October 2015; published 13 January 2016)

\begin{abstract}
Despite relevance to disparate areas such as cloud microphysics and tribology, major gaps in the understanding of the structures and phase transitions of low-dimensional water ice remain. Here, we report a first principles study of confined 2D ice as a function of pressure. We find that at ambient pressure hexagonal and pentagonal monolayer structures are the two lowest enthalpy phases identified. Upon mild compression, the pentagonal structure becomes the most stable and persists up to $\sim 2 \mathrm{GPa}$, at which point the square and rhombic phases are stable. The square phase agrees with recent experimental observations of square ice confined within graphene sheets. This work provides a fresh perspective on 2D confined ice, highlighting the sensitivity of the structures observed to both the confining pressure and the width.
\end{abstract}

DOI: 10.1103/PhysRevLett.116.025501

Confined and interfacial water ice is ubiquitous in nature, playing an important role in a wide range of areas such as rock fracture, friction, and nanofluidics [1-4]. As a result of a delicate balance of forces (hydrogen bonding, van der Waals forces, and interaction with the confining material or substrate) confined and interfacial water forms a rich variety of structures [5-10]. Almost every specific system examined has revealed a different structure such as a 2D overlayer built from heptagons and pentagons on a platinum surface or the square ice observed within layers of graphene $[8,10]$. This shows that, in contrast to bulk ice, the phase behavior of 2D ice is much less well understood.

From an experimental perspective, a full exploration of the phase diagram of $2 \mathrm{D}$ ice has not been achieved yet. However, recent experiments revealed the exciting possibility of exploring 2D ice structures at specific conditions by trapping water within layered materials [2,10,11]. For example, by confining water between layers of graphene, it is possible to create so-called nanocapillaries in which water experiences a pressure in the gigapascal regime due to the van der Waals forces pulling the sheets together [10]. Using transmission electron microscopy (TEM), square ice structures from a single up to a few layers were observed in such graphene nanocapillaries [10]. Force field simulations performed as part of the same study of confined water in graphene layers reproduced some aspects of the experiments, such as the square monolayer ice structure. Indeed,

Published by the American Physical Society under the terms of the Creative Commons Attribution 3.0 License. Further distribution of this work must maintain attribution to the author(s) and the published article's title, journal citation, and DOI. prior to this recent study, there was already a long tradition of computer simulation studies of nanoconfined water, mostly involving classical force field approaches [12-27]. Such work has been incredibly valuable and has provided considerable insight into the structures and phase transitions of monolayer and multilayer ice. The 2D ice structures predicted include hexagonal, pentagonal, quasicrystalline, hexatic, and orthogonal phases as well as various amorphous structures. Although the relative stability of the structures depends on the particular force field used, for monolayer ice an orthogonal phase has been widely predicted to be stable across a broad pressure regime $[13,14,19,23]$. Given the sensitivity of force field studies of confined ice to the potential used and the aspiration to understand the observation of square ice, a systematic study with an electronic structure method such as density functional theory (DFT) is highly desirable. DFT studies of ice do not come without their own sensitivity to the exchange-correlation functional used; however, they have proved to be very useful in predicting and understanding structures of adsorbed water and bulk ice [5-9,28].

Here, we report a systematic study of 2D phases of water ice from first principles based on an unbiased exploration of monolayer ice structures. The much greater computational cost of DFT compared to force field methods means that we cannot currently map the entire phase diagram of monolayer confined water. Instead, we focus on the phase transitions as a function of lateral pressure and confinement width at $0 \mathrm{~K}$. The stable structures identified at different pressures include a hexagonal structure, a pentagonal Cairo tiling (CT) structure, a flat square structure, and a buckled rhombic structure. The observation of a flat square structure is consistent with the recent experimental observation. 
However, the sequence of low energy phases identified differs significantly from that predicted in force field based studies [13,14,19,21,23] and a recent DFT report [26]. Interestingly, the sequence of structures observed depends sensitively on the confinement width used, suggesting that it should be possible to tune the monolayer ice structures produced in experiments by, e.g., varying the confining material.

In order to explore ice structures in an unbiased manner, we used the $a b$ initio random structure search technique $[29,30]$, an approach which has previously predicted new ice, 2D, and interfacial structures [31,32]. Structures from ambient up to a lateral pressure of $10 \mathrm{GPa}$ were considered. Periodic boundary conditions are used with unit cells containing $2,4,6,8,10,12,18$, and 24 water molecules [33]. The 2D confinement was introduced via a Morse potential fit to quantum Monte Carlo results for the binding of a water monomer to graphene [34]. By tuning the confinement width, we are able to not only study the general properties of water under flat and smooth confinement but also compare it with the recent experiments of ice in graphene confinement [10]. Our electronic structure calculations were carried out using the Vienna $a b$ initio simulation package (VASP) [35] with the DFT + van der Waals approach [36] in conjunction with the PerdewBurke-Ernzerhof exchange-correlation functional [37]. Tests with other exchange-correlation functionals show that, while the transition pressures between the various phases depend to some extent on the choice of exchangecorrelation functional, the overall conclusions do not change. See the Supplemental Material [38] for these results as well as further computational details and tests of 2D ice confined within actual sheets of graphene.

From a preliminary set of calculations, we established that the optimal separation between graphene sheets in which a monolayer of water is sandwiched is somewhere between 6.0 and $6.5 \AA$ (see Fig. S3 in the Supplemental Material [38]). With this in mind, we first report results for water within smooth confining potentials that are either 6.0 or $6.5 \AA$ wide. With such confinement, two phases have been identified at ambient pressure which have exceedingly similar enthalpies. These are a hexagonal monolayer structure, resembling a hexagonal bilayer, and a Cairo-tiled structure built exclusively from water pentagons. With the particular exchange-correlation functional used, the less dense hexagonal structure is marginally more stable than the pentagonal structure by $5 \mathrm{meV} / \mathrm{H}_{2} \mathrm{O}$. This difference drops to just $2 \mathrm{meV} / \mathrm{H}_{2} \mathrm{O}$ when harmonic zero point energy effects are taken into account (see Table S2 and Fig. S11 in the Supplemental Material [38]). Tests with other exchange-correlation functionals generally concur that the energy difference between the two phases is tiny, with a vanishingly small preference for the hexagonal structure (see Table S2 in the Supplemental Material [38]). Anharmonic zero point energy differences between the two phases and finite temperature effects could also easily exceed the energy difference [49], suggesting that both the hexagonal and CT phases could be observed at ambient pressures.

The hexagonal structure has $p 6 \mathrm{~mm}$ wallpaper group symmetry if only oxygen atoms are considered and is built exclusively of six-membered rings [see Fig. 1(a)]. Water molecules are threefold coordinated, with half of them having one $\mathrm{OH}$ bond directed out of the monolayer (a so-called dangling $\mathrm{OH}$ ). The average $\mathrm{O}-\mathrm{O}$ separation in this monolayer hexagonal phase is about $2.72 \AA$, which is similar to the bulk O-O separation in ice I [50]. However, the confined hexagonal structure identified here is quite flat, with the vertical separation between oxygen atoms $<0.3 \AA$, much smaller than the $0.9 \AA$ buckling within a hexagonal layer in bulk ice I [51]. Given that bulk ice I is built from hexagonal layers and that double-layer hexagonal structures have been observed frequently in force field simulations [16-18], it is not surprising that a low enthalpy hexagonal structure should be identified. However, monolayer hexagonal ice has yet to be observed experimentally, and in force field simulations it has only been found when a hexagonally patterned substrate has been used as a template [21].

The pentagonal structure identified has a wallpaper group symmetry of $p 4 g m$ [see Fig. 1(b)]. The unit cell has 12 water molecules, four with dangling $\mathrm{OH}$ bonds.
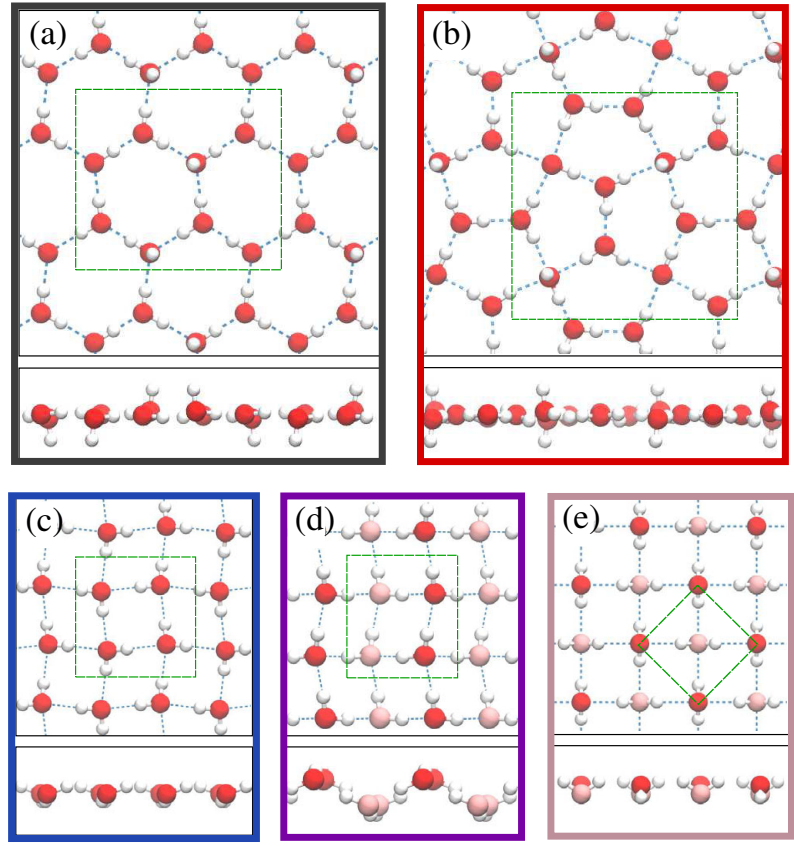

FIG. 1. Monolayer ice structures. The top and side views of (a) the hexagonal, (b) the Cairo tiling (CT), (c) the flat square (f-SQ), (d) the rhombic (b-RH), and (e) the buckled square (b-SQ) structures. Red and pink spheres represent oxygen atoms at different heights, and the white spheres are hydrogen atoms. The green boxes show the primitive unit cells. 
One-third of the water molecules are fourfold coordinated and the rest are threefold coordinated. The higher average coordination and smaller ring size of the $\mathrm{CT}$ phase renders the density of this phase higher than that of the hexagonal phase (see Figs. S5 and S6 in the Supplemental Material [38]). Therefore, upon increasing the pressure the CT phase clearly becomes more stable than the hexagonal phase. After searching for the lowest enthalpy structures at finite pressure we find that the CT structure is clearly the most stable in a broad range of pressures all the way up to $\sim 2 \mathrm{GPa}$ (see Fig. 2). The stability regime of the monolayer $\mathrm{CT}$ phase and the small energy difference at ambient pressure suggest that five-membered rings are more important in 2D ice than in 3D ice, where they only appear in the 0.2-0.7 GPa range (in ices III, V, IX, and XIII) [52]. There is some precedent for the pentagonal based structure being proposed here. First and foremost, it is a monolayer version of the double-layer confined ice structure identified in Ref. [17] on the basis of simulations with a coarse grained model of water. In addition, a 1D pentagonal ice structure has been observed with scanning tunneling microscopy on a metal surface, although the structure of the 1D pentagonal chain is very different from $2 \mathrm{D}$ pentagonal ice [6]. The only

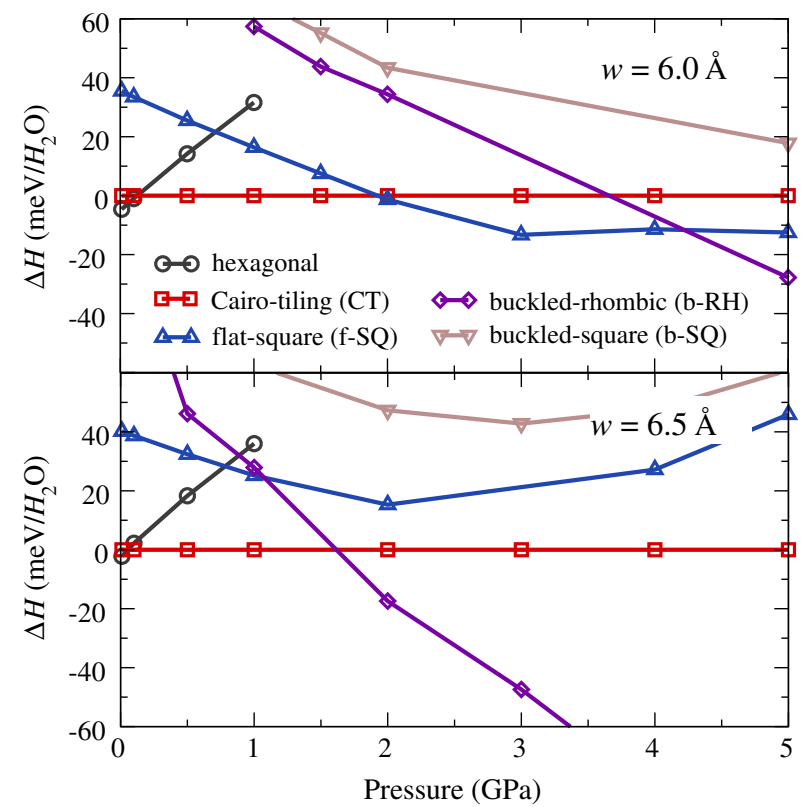

FIG. 2. Enthalpies of the water monolayer phases as a function of lateral pressure under (top panel) 6.0 and (bottom panel) $6.5 \AA$ confinement. $\Delta H$ is the relative enthalpy with respect to the CT phase. Enthalpy $H=E_{\text {water }}+E_{\text {confinement }}+P \times A \times h$, where $E_{\text {water }}$ is the total energy per water molecule, $E_{\text {confinement }}$ is the energy (per water molecule) in the confinement potential, $A$ is the lateral area per water molecule, $w$ is the width of confinement, $P$ is the lateral pressure, $P=\frac{1}{2}\left(\sigma_{x x}+\sigma_{y y}\right)$, $\sigma=\sigma^{\prime} \times L_{z} / h, \sigma_{x x}^{\prime}$ and $\sigma_{y y}^{\prime}$ are the calculated lateral diagonal stress tensor elements for the slab-vacuum model, $L_{z}$ is the length of the cell in the out-of-plane direction, and the layer height $h$ equals the width of the confinement $w$ [54]. purely $2 \mathrm{D}$ structure similar to our prediction that we are aware of is a recently proposed allotrope of carbon [53].

Previous force field studies have suggested that a rhombic phase is the most stable at ambient pressures $[13,14]$. Our own force field studies with either TIP4P/2005 [55] or SPC/E [56] indeed find that the rhombic structure has the lowest enthalpy (see Fig. S10 in the Supplemental Material [38]). However, with DFT it is considerably less stable than the hexagonal and CT structures. Similarly, a recent DFT study concluded that a square structure is more stable than any hexagonal structure [26]. Here, we find that our most stable square structure has a higher enthalpy by $43 \mathrm{meV} / \mathrm{H}_{2} \mathrm{O}$ than the hexagonal phase at ambient pressure. In the Supplemental Material [38] we trace this difference to the different computational setups. As shown in the Supplemental Material [38], we are confident that the hexagonal and CT monolayer ice structures are indeed more stable than any square ice structure at the low pressure limit.

At pressures beyond $2 \mathrm{GPa}$, higher density phases obeying the Bernal-Fowler-Pauling ice rules are identified more frequently in the structure searches. This includes a flat square phase (f-SQ) and a buckled rhombic phase (b-RH). Both phases consist of fourfold coordinated water molecules with two donor hydrogen bonds (HBs) and two acceptor HBs. The f-SQ structure has a $p 4 g m$ wallpaper group symmetry where the dipoles of the water molecules are distributed on two orthogonal antiferroelectric sublattices [see Fig. 1(c)]. The HB network of the b-RH phase is similar to f-SQ, but it is buckled and has a higher lateral density [see Fig. 1(d)]. The relative stability of the f-SQ and b-RH structures in the 2-4 GPa regime depends sensitively on the confinement width (see Fig. 2). At $6.0 \AA \mathrm{f}$-SQ is more stable, while at $6.5 \AA \mathrm{b}$-RH has the lower enthalpy. Beyond $4 \mathrm{GPa}$ the b-RH phase is more stable than any other structure identified. Several other metastable structures belonging to the $\mathrm{b}-\mathrm{RH}$ family with different hydrogen bond ordering have also been observed. However, since a more delicate discussion of hydrogen ordering is beyond the scope of this Letter, we only show one of the most stable members of the b-RH family at the pressure and confinement conditions considered [see Fig. 1(d)].

We also identified a second metastable square phase which we dub "b-SQ" because of its buckled "basketweavelike" pattern of HBs [see Fig. 1(e)]. Its lattice structure resembles a 2D projection of bulk ice VIII, but it is unique in that the two sublayers are hydrogen bonding with each other. The energy of the b-SQ phase is higher than the most stable phases identified; however, because of its unique hydrogen bonding arrangement and as it might be possible to observe it in systems where the substrate has a square lattice, we feel it is worth reporting.

Beyond the phase behavior of monolayer ice confined within the 6.0 to $6.5 \AA$ regime, we also explored a broader range of confinement widths at different pressures. These 
additional calculations support the validity of the conclusions reached but also show that there is scope for altering the relative enthalpies of the various phases by tuning the confinement. The phase diagram for monolayer water with respect to lateral pressure (0-10 $\mathrm{GPa})$ and confinement width $(5-8 \AA)$ that emerges from these calculations is shown schematically in Fig. 3. At small confinements and low pressures $(<\sim 0.1 \mathrm{GPa})$, the hexagonal and $\mathrm{CT}$ phases are preferred. Increasing the pressure at small confinement widths results in a sequence of phase transitions from the pentagonal to the square and rhombic phases. For larger confinement widths, the b-RH phase is generally favored.

The f-SQ phase is found to be stable in the $2-4 \mathrm{GPa}$ range at $6.0 \AA$ confinement. The structure of the f-SQ phase and the approximate pressure at which it appears are consistent with the recent experimental observation of a square ice phase in graphene nanocapillaries [10]. This lends some support to the predictions made here and suggests that it might also be possible to observe the other structures predicted by, for example, controlling the density of water inside the nanocapillaries or with another 2D material with lower interlayer adhesion. In addition to TEM, which has already been used, techniques such as scanning tunneling microscopy and atomic force microscopy might be able to further substantiate 2D ice lattices and hydrogen ordering in the future [57,58]. In Fig. S6 in the Supplemental Material we also show that different 2D ice monolayer structures have quite different vibrational properties [38]. For example, the hexagonal and the CT phase have vibrational modes around $3700 \mathrm{~cm}^{-1}$ due to

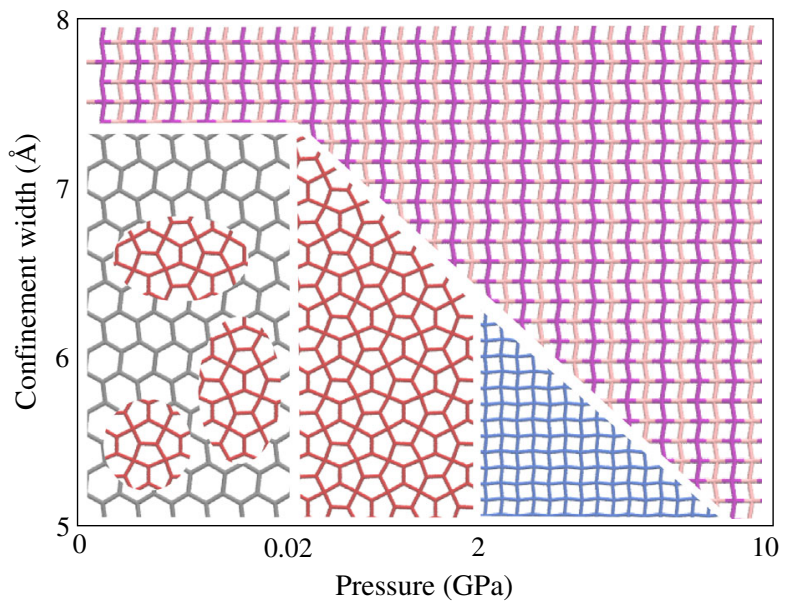

FIG. 3. Sketch of the phase diagram of monolayer ice with respect to lateral pressure and confinement width. For clarity, the pressure axis has an artificial scale with the various pressures indicated corresponding to the transition pressures obtained at $6 \AA$ confinement. Note that at ambient pressure the enthalpies of the hexagonal and Cairo-tiled phases are essentially degenerate, but by $\sim 0.02 \mathrm{GPa}$ the Cairo-tiled phase is more clearly favored. See Fig. S8 in the Supplemental Material [38] for more results of enthalpies with respect to confinement width. dangling $\mathrm{OH}$ groups and lower frequency stretching modes $<3000 \mathrm{~cm}^{-1}$ arising from strong HBs. The stretching regions also have quite different total widths for the different phases, and the bending mode of the b-SQ phase is softer than the other phases by $\sim 120 \mathrm{~cm}^{-1}$. Therefore, it should also be possible to discriminate one phase from another with vibrational spectroscopy.

In summary, monolayer ice phases and their phase transitions in confinement have been studied with DFT and a random structure search approach. At ambient pressure, we have predicted hexagonal and pentagonal Cairo-tiled structures, which are similar in enthalpy and more stable than other structures. The CT structure becomes more stable than the hexagonal structure when lateral pressure is applied. Upon increasing the pressure to above about $2 \mathrm{GPa}$, high density square and rhombic phases are observed. Looking forward, a complete description of the phase diagram of $2 \mathrm{D}$ water is desirable. Experimentally, it would be interesting to explore a broader range of temperatures, water densities, and confining materials. From the computational perspective, there may be yet more interesting phases of 2D ice to find in the future with larger unit cells, in the multilayer regime and through tuning the confining potential. It would also be desirable to explore 2D ice at finite temperatures with electronic structure methods, such as $a b$ initio molecular dynamics. In addition, in light of the small ring sizes and relatively short intermolecular separations, 2D ice also provides further opportunities for investigating collective proton quantum dynamics [59-61].

J. C. and A. M. are supported by the European Research Council under the European Union's Seventh Framework Programme (FP/2007-2013)/ERC Grant Agreement No. 616121 (HeteroIce project). A. M is also supported by the Royal Society through a Royal Society Wolfson Research Merit Award. C. J. P. and G. S. are supported by EPSRC Grants No. EP/G007489/2 and No. EP/J010863/2. C. G. S. is supported by the Royal Society (Grant No. UF100144). We are also grateful to the London Centre for Nanotechnology for their computational resources, UCL Research Computing, and to the UKCP consortium (Grant No. EP/F036884/1) for access to Archer.

*ji.chen@ucl.ac.uk

angelos.michaelides@ucl.ac.uk

[1] D. Chandler, Nature (London) 437, 640 (2005).

[2] K. Xu, P. Cao, and J. R. Heath, Science 329, 1188 (2010).

[3] J. Carrasco, A. Hodgson, and A. Michaelides, Nat. Mater. 11, 667 (2012)

[4] Y. Gao, S. Kim, S. Zhou, H.-C. Chiu, D. Nlias, C. Berger, W. deHeer, L. Polloni, R. Sordan, A. Bongiorno, and E. Riedo, Nat. Mater. 14, 714 (2015).

[5] J. Yang, S. Meng, L. F. Xu, and E. G. Wang, Phys. Rev. Lett. 92, 146102 (2004). 
[6] J. Carrasco, A. Michaelides, M. Forster, S. Haq, R. Raval, and A. Hodgson, Nat. Mater. 8, 427 (2009).

[7] M. Forster, R. Raval, A. Hodgson, J. Carrasco, and A. Michaelides, Phys. Rev. Lett. 106, 046103 (2011).

[8] S. Nie, P. J. Feibelman, N. C. Bartelt, and K. Thürmer, Phys. Rev. Lett. 105, 026102 (2010).

[9] J. Chen, J. Guo, X. Meng, J. Peng, J. Sheng, L. Xu, Y. Jiang, X.-Z. Li, and E.-G. Wang, Nat. Commun. 5, 4056 (2014).

[10] G. Algara-Siller, O. Lehtinen, F. C. Wang, R. R. Nair, U. Kaiser, H. A. Wu, A. K. Geim, and I. V. Grigorieva, Nature (London) 519, 443 (2015).

[11] Q. Li, J. Song, F. Besenbacher, and M. Dong, Acc. Chem. Res. 48, 119 (2015).

[12] K. Koga, H. Tanaka, and X. C. Zeng, Nature (London) 408, 564 (2000).

[13] R. Zangi and A. E. Mark, Phys. Rev. Lett. 91, 025502 (2003).

[14] K. Koga and H. Tanaka, J. Chem. Phys. 122, 104711 (2005).

[15] N. Giovambattista, P. J. Rossky, and P. G. Debenedetti, Phys. Rev. Lett. 102, 050603 (2009).

[16] S. Han, M. Y. Choi, P. Kumar, and H. E. Stanley, Nat. Phys. 6, 685 (2010).

[17] J. C. Johnston, N. Kastelowitz, and V. Molinero, J. Chem. Phys. 133, 154516 (2010).

[18] N. Kastelowitz, J. C. Johnston, and V. Molinero, J. Chem. Phys. 132, 124511 (2010).

[19] J. Bai, C. A. Angell, and X. C. Zeng, Proc. Natl. Acad. Sci. U.S.A. 107, 5718 (2010).

[20] J. Bai and X. C. Zeng, Proc. Natl. Acad. Sci. U.S.A. 109, 21240 (2012).

[21] A. L. Ferguson, N. Giovambattista, P. J. Rossky, A. Z. Panagiotopoulos, and P. G. Debenedetti, J. Chem. Phys. 137, 144501 (2012).

[22] N. Giovambattista, P. Rossky, and P. Debenedetti, Annu. Rev. Phys. Chem. 63, 179 (2012).

[23] W.-H. Zhao, J. Bai, L.-F. Yuan, J. Yang, and X. C. Zeng, Chem. Sci. 5, 1757 (2014).

[24] W.-H. Zhao, L. Wang, J. Bai, L.-F. Yuan, J. Yang, and X. C. Zeng, Acc. Chem. Res. 47, 2505 (2014).

[25] Q. Lu, J. Kim, J. D. Farrell, D. J. Wales, and J. E. Straub, J. Chem. Phys. 141, 18C525 (2014).

[26] F. Corsetti, P. Matthews, and E. Artacho, arXiv:1502.03750.

[27] F. Corsetti, J. Zubeltzu, and E. Artacho, arXiv:1506.04668.

[28] G. A. Tribello, B. Slater, and C. G. Salzmann, J. Am. Chem. Soc. 128, 12594 (2006).

[29] C. J. Pickard and R. J. Needs, Phys. Rev. Lett. 97, 045504 (2006).

[30] C. J. Pickard and R. J. Needs, J. Phys. Condens. Matter 23, 053201 (2011).

[31] C. J. Pickard, M. Martinez-Canales, and R. J. Needs, Phys. Rev. Lett. 110, 245701 (2013).

[32] G. Schusteritsch and C. J. Pickard, Phys. Rev. B 90, 035424 (2014).

[33] The most stable structures observed contain 8 (hexagonal), 12 (CT), 4 (f-SQ), and 4 (b-RH) water molecules in each unit cell. Upon increasing the number of molecules in the cell to 18 and 24 , we did not find any new low energy structures.

[34] J. Ma, A. Michaelides, D. Alfè, L. Schimka, G. Kresse, and E. Wang, Phys. Rev. B 84, 033402 (2011).
[35] G. Kresse and J. Furthmüller, Phys. Rev. B 54, 11169 (1996).

[36] A. Tkatchenko and M. Scheffler, Phys. Rev. Lett. 102, 073005 (2009).

[37] J. P. Perdew, K. Burke, and M. Ernzerhof, Phys. Rev. Lett. 77, 3865 (1996).

[38] See Supplemental Material at http://link.aps.org/ supplemental/10.1103/PhysRevLett.116.025501, which includes Refs. [39-48], for more details on our calculations, additional information on the ice phases, and various tests.

[39] G. Kresse and D. Joubert, Phys. Rev. B 59, 1758 (1999).

[40] J. Heyd, G. E. Scuseria, and M. Ernzerhof, J. Chem. Phys. 118, 8207 (2003).

[41] M. Dion, H. Rydberg, E. Schröder, D. C. Langreth, and B. I. Lundqvist, Phys. Rev. Lett. 92, 246401 (2004).

[42] J. Heyd, G. E. Scuseria, and M. Ernzerhof, J. Chem. Phys. 124, 219906 (2006).

[43] J. Klimeš, D. R. Bowler, and A. Michaelides, J. Phys. Condens. Matter 22, 022201 (2010).

[44] J. Klimeš, D. R. Bowler, and A. Michaelides, Phys. Rev. B 83, 195131 (2011).

[45] B. Santra, J. Klimeš, D. Alfè, A. Tkatchenko, B. Slater, A. Michaelides, R. Car, and M. Scheffler, Phys. Rev. Lett. 107, 185701 (2011).

[46] E. D. Murray and G. Galli, Phys. Rev. Lett. 108, 105502 (2012).

[47] F. Corsetti, M.-V. Fernández-Serra, J. M. Soler, and E. Artacho, J. Phys. Condens. Matter 25, 435504 (2013).

[48] J. Wang, G. Román-Pérez, J. M. Soler, E. Artacho, and M.-V. Fernández-Serra, J. Chem. Phys. 134, 024516 (2011).

[49] E. A. Engel, B. Monserrat, and R. J. Needs, Phys. Rev. X 5, 021033 (2015).

[50] K. Röttger, A. Endriss, J. Ihringer, S. Doyle, and W. F. Kuhs, Acta Crystallogr. Sect. B 50, 644 (1994).

[51] V. F. Petrenko and R. W. Whitworth, Physics of Ice (Oxford University Press, New York, 2002).

[52] C. G. Salzmann, P. G. Radaelli, B. Slater, and J. L. Finney, Phys. Chem. Chem. Phys. 13, 18468 (2011).

[53] S. Zhang, J. Zhou, Q. Wang, X. Chen, Y. Kawazoe, and P. Jena, Proc. Natl. Acad. Sci. U.S.A. 112, 2372 (2015).

[54] In the calculation, $\sigma_{x x}^{\prime} \times L_{z}$ and $\sigma_{y y}^{\prime} \times L_{z}$ are conserved quantities and the calculation of the enthalpy is independent of the definition of $h$. Therefore, the shape of the phase diagram would not be affected by the definition. However, the definition of $h$ does affect the values of the transition pressures predicted at high pressures. Our definition indicates an upper limit of layer height (width of confinement), corresponding to a lower limit of transition pressures. Assuming the lower limit of width is around $w / 2$, the upper limit of the transition pressures are two times what we have reported. The values of the phase transition pressure are also affected by the accuracy of the DFT methods used, as discussed in the Supplemental Material [38].

[55] C. Vega, J. L. F. Abascal, M. M. Conde, and J. L. Aragones, Faraday Discuss. 141, 251 (2009).

[56] H. J. C. Berendsen, J. R. Grigera, and T. P. Straatsma, J. Phys. Chem. 91, 6269 (1987).

[57] J. Zhang, P. Chen, B. Yuan, W. Ji, Z. Cheng, and X. Qiu, Science 342, 611 (2013). 
[58] J. Guo, X. Meng, J. Chen, J. Peng, J. Sheng, X.-Z. Li, L. Xu, J.-R. Shi, E. Wang, and Y. Jiang, Nat. Mater. 13, 184 (2014).

[59] X.-Z. Li, M. I. J. Probert, A. Alavi, and A. Michaelides, Phys. Rev. Lett. 104, 066102 (2010).
[60] C. Drechsel-Grau and D. Marx, Phys. Rev. Lett. 112, 148302 (2014).

[61] X. Meng, J. Guo, J. Peng, J. Chen, Z. Wang, J.-R. Shi, X.-Z. Li, E.-G. Wang, and Y. Jiang, Nat. Phys. 11, 235 (2015). 